\title{
Indagando en Sant Antoni (Barcelona) en torno a la relación de la bicicleta con la gentrificación
}

\author{
À. Cebollada (a), A. Sillero (b), E. Anaya (c) \\ (a) Departamento de Geografía, Universitat Autònoma de Barcelona, angel.cebollada@uab.cat \\ (b) Departamento de Geografía, Universitat Autònoma de Barcelona, azahara.sillero@uab.cat \\ (c) Centre for Environmental Policy, Imperial College London, e.anaya14@imper-ial.ac.uk
}

\begin{abstract}
Resumen
Estamos asistiendo a un profundo proceso de cambio social y económico en las grandes ciudades, o al menos en determinados barrios de las mismas. Las dinámicas de un mercado altamente desregularizado junto con los procesos de globalización llevan a que en determinadas zonas urbanas se den procesos de sustitución de sus residentes por otros de más poder adquisitivo y/o por turistas. En esta comunicación queremos abordar el rol que pueda tener la bicicleta en estos procesos de cambio social y económico asumiendo que los nuevos residentes interpretan la presencia y el uso de este modo de transporte como indicador de mejor calidad urbana. La investigación se ha realizado combinando metodologías cuantitativas y cualitativas.
\end{abstract}

Palabras clave: Bicicleta, gentrificación, Barcelona, cambio social, turismo.

\section{INTRODUCCIÓN. LAS CIUDADES EN LA ECONOMÍA GLOBAL}

En los últimos decenios se han producido importantes cambios económicos y sociales en nuestras ciudades. Estos cambios no se explican solamente por elementos endógenos, sino que se producen principalmente como resultado de un mercado global altamente desregularizado. Capitales internacionales fijan sus objetivos en determinados barrios para clientes internacionales de segmentos de rentas altas. Además, las nuevas tecnologías permiten la aparición de un trabajo en red protagonizado por los denominados nómadas digitales, que deslocalizan absolutamente su lugar de residencia por el de la empresa en la que trabajan. Y todavía, la turificación de las ciudades contribuye a estos cambios. Estos visitantes, generan la demanda de nuevos productos, nuevos comercios, nuevas formas de vida y asimismo introducen nuevos símbolos. Es este capítulo nos planteamos qué rol asume la bicicleta en este proceso de cambio y qué simboliza.

\section{EL ÁREA DE ESTUDIO. SANT ANTONI: UN BARRIO EN TRANSFORMACIÓN}

Sant Antoni es un barrio del Eixample barcelonés, en el sector suroriental. Morfológicamente tiene forma triangular y se encaja entre la Barcelona preindustrial (Ciutat Vella) y el barrio del Poble-sec, en la ladera de la montaña de Montjuïc. Su superficie es de 0,8 km2 y la población de 38.033 habitantes (apenas un 2,4\% de la población del municipio). Se considera uno de los barrios tradicionales de la ciudad, con un sentimiento identitario diferenciado de los adyacentes.

En los últimos años, Sant Antoni está en el foco del debate público debido al proceso de cambio socioeconómico que se está produciendo. La alta proliferación de bares en torno a Carrer Parlament es un ejemplo de ello. A principios de 2017, un artículo en la revista cultural Time Out lo calificaba como "el barrio de moda de Barcelona" por el reciente aumento de "locales nuevos que conviven con los de toda la vida" (Time Out, 2017). Entre los ejemplos de locales a visitar, la revista recomienda varios talleres de motos antiguos hoy restaurados y transformados en bares. Su tradicional mercado dominical de libros ha acentuado el carácter popular y en cierta medida bohemio del barrio. Como la otra cara de la misma moneda, a finales del mismo año, la plataforma ciudadana Fem Sant Antoni sacaba a la luz el Mapa de la Gentrificación, donde señalaba la existencia de 46 bares en sólo 
tres islas de casas que dificultan la capacidad de los vecinos/as de poder hacer vida de barrio (Fem Sant Antoni, 2017).

Pero el proceso de cambio no ha hecho más que empezar. Según los indicios, existen dos actuaciones -ya en marcha- que pueden catapultar al barrio hacia la transformación definitiva. Por un lado, el Mercado de Sant Antoni, fundado en 1882 y centro neurálgico del barrio, ha vivido en obras durante casi diez años, ha vivido abriéndose finalmente al público en mayo de 2018... Teniendo en cuenta las experiencias previas de rehabilitación de mercados en Barcelona, como la rehabilitación del Mercado de Santa Caterina, el miedo a la subida del alquiler en la zona se ha expandido, y en el barrio no hay quien no haya oído la palabra gentrificación. Por otro lado, a principios de 2017 se impulsaba la propuesta de una nueva superilla (supermanzana) en el entorno del citado mercado, que pretende recuperar más de $26.000 \mathrm{~m}^{2}$ para los viandantes (Ajuntament de Barcelona, sin fecha) a través de una operación de pacificación del tránsito. La operación urbanística que se ha iniciado en los entornos del mercado incluye otras manzanas de los alrededores y se prevé que finalice en la primavera de 2019. El proyecto también contempla el incremento del verde urbano.Se intuye que estas operaciones culminarán un cambio sociodemográfico importante ya observable en la zona. Una mezcla de interés por parte de personas de rentas superiores, junto el aumento del turismo (y la consecuente aparición de la ocupación de viviendas para usos estacionales), así como la compra de viviendas como mecanismo de inversión del mercado inmobiliario internacional, han puesto en riesgo la capacidad de los actuales residentes de poder continuar habitando el barrio.
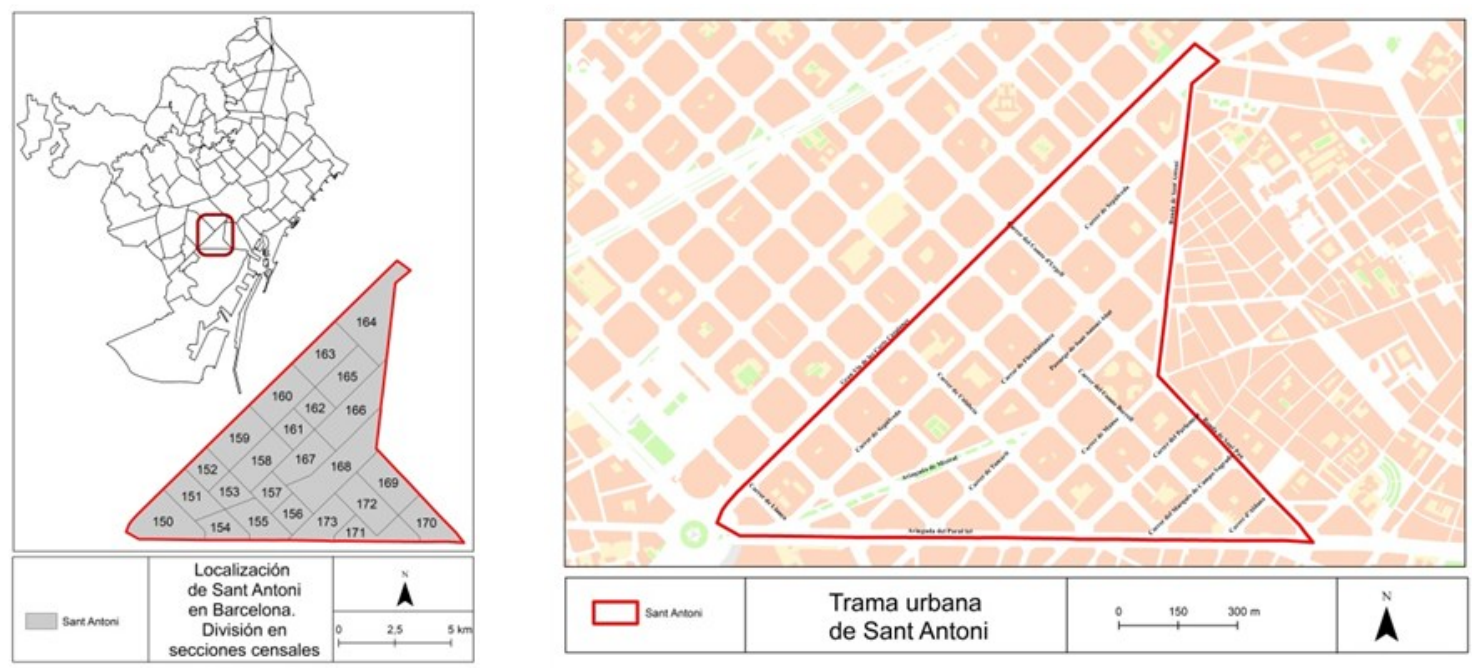

Figura 1.

Fuente: Elaboración propia.

\section{LA RELACIÓN ENTRE LA BICICLETA Y LA GENTRIFICACIÓN}

La elección de Sant Antoni para la realización de este estudio responde a la vinculación del mismo con un proyecto más amplio que se dedica a estudiar el proceso de cambio social, económico y urbano del barrio, así como los efectos de la gentrificación. En lo concreto, el interés de esta comunicación y del estudio que aún está en curso, se centra en el papel que juega la bicicleta en la transformación del barrio.

El concepto de gentrificación fue originalmente acuñado por Ruth Glass en 1964. Desde entonces, este proceso se ha vuelto mucho más complejo, especialmente en su conexión con las nuevas dinámicas de acumulación de capital vinculadas a la globalización. Es, lo que se ha llamado, la tercera ola de la gentrificación (Chan, 2014).

Dentro de las múltiples combinaciones y matices que se presentan, se ha comprobado que gran parte de la producción académica que se encarga de estudiar la relación entre la bicicleta (a nivel general) y la gentrificación, recae sobre estudios de caso en América del Norte: desde el estudio comparativo de Toronto, Montreal y Vancouver (Danyluk y Ley, 2007) pasando por el estudio de diferentes ciudades de los Estados Unidos tales como Nueva York (Stein, 2011), Washington DC (Gibson, 2013), San Francisco (Stehlin, 2014), Portland y Chicago (Flanagan, Lachapelle, y El-geneidy, 2016), Seattle y Los Ángeles (Lugo, 2017) ó Boston (Vith y Mössner, 2017). Es un fenómeno de creciente interés desde principios de la entrada del siglo XXI, vinculado al resurgir de la bici- 
cleta en la ciudad y a la expansión de los procesos de gentrificación que acarrea el urbanismo neoliberal (Smith, 2002).

No es mera coincidencia: el aumento de las investigaciones académicas en esta área geográfica podría explicarse porque según Vith y Mössner (2017) la introducción de infraestructura bici en Estados Unidos se suele dar, de manera frecuente, en barrios o bien que están viviendo un proceso de gentrificación o bien que han sido recientemente gentrificados. Además, la promoción de la bicicleta como forma de transporte, así como la inversión en infraestructura ciclista en la ciudad se ha defendido comúnmente bajo un discurso vinculado al desarrollo económico (Stehlin, 2014; Vith y Mössner, 2017). Es por esto que muchos de los artículos se centran en la correlación entre la inversión en infraestructura ciclista con los fenómenos de gentrificación, o la reproducción de la desigualdad a través de la implantación de la infraestructura en barrios ya acomodados.

Por otro lado, los estudios de gentrificación y movilidad no sólo recaen sobre el impacto de la infraestructura en el propio cambio, sino en los aspectos culturales que llevan consigo los procesos de gentrificación. Es decir, los nuevos habitantes, o las nuevas clases sociales, que -según la teoría- llegan a estos barrios gentrificados, representan y traen consigo un estilo de vida cosmopolita y promovedor de la conciencia medioambiental que, acorde con nuestro estudio, encaja con la bicicleta como símbolo y modo de desplazamiento urbano (Stehlin, en Vith y Mössner, 2017). En nuestro estudio, así como lo hacen Vïth y Mössner (2017), hemos tratado de estudiar la relación de la bicicleta en Sant Antoni con los dos discursos predominantes: por un lado la desigualdad de la infraestructura y por el otro la apropiación de la bicicleta por parte de las clases creativas.

\section{METODOLOGÍA}

Para ello, en primer lugar, se ha realizado un estudio tentativo de las características sociodemográficas de la zona para observar qué datos cuantitativos evidencian la transformación que se está dando en Sant Antoni. También se ha recogido información mediante el trabajo de campo con observaciones (cambios físicos) y entrevistas exploratorias (percepción del cambio). Por último, se han elegido tres plataformas que están vinculadas al cambio cultural en los barrios gentrificados de Barcelona tales como Airbnb, Donkey Republic, así como la iniciativa local The Trend Map.

El análisis sociodemográfico del barrio se ha realizado a partir de los datos existentes de estadísticas oficiales centralizadas por el departamento de estadística de Barcelona que a su vez son de fuentes distintas, según cada variable. La escala territorial trabajada han sido las secciones censales. El barrio de Sant Antoni se divide en 24 secciones censales. En este caso se han utilizado los datos demográficos y espaciales básicos y las variables socioeconómicas que utiliza el mismo ayuntamiento de la ciudad para la realización del indicador indirecto de Renta Familiar Disponible por barrios. En concreto, se han utilizado en esta comunicación: el porcentaje de población con estudios superiores en relación con la población mayor de 25 años, el valor catastral de los locales, el porcentaje de vehículos según población total y el ratio de vehículos de más de $16 \mathrm{CV}$ de potencia fiscal según el número total de vehículos. Además, se han tomado los datos de precios de venta por $\mathrm{m}^{2}$ de la vivienda de segunda mano, en esta ocasión por barrios de la ciudad (73 en total). Los datos sobre la oferta de bicicleta provienen del Open Data del mismo ayuntamiento. Todos estos datos han sido incorporados a un Sistema de Información Geográfico para su análisis espacial y algunas de estas representaciones cartográficas han sido incorporadas a la comunicación.

Con el fin de complementar los datos cuantitativos, se han realizado diferentes salidas y observaciones a lo largo de los meses de febrero, marzo y abril para documentar el papel de la bicicleta en este proceso de cambio.

Se han localizado las tiendas existentes en el barrio vinculadas a la venta o alquiler de bicicletas para poder estudiar el tipo de negocio y sus orígenes. En los locales que ha sido posible, se han realizado entrevistas in situ y exploratorias a los trabajadores o dueños del local.

Por otro lado, para estudiar la vinculación de la bicicleta como reclamo en esta etapa de reurbanización, se ha estudiado:

- La oferta actual de bicicletas de la empresa Donkey Republic en el barrio. Para esto se realizó la descarga de la app de la compañía y se revisó la oferta de aparcamientos durante una semana en tres horas distintas del día.

- $\quad$ Los negocios considerados como trendy (de moda) y su posible vinculación con la bicicleta. Para ello se ha revisado la plataforma The Trend Map, que se dedica a catalogar bares (hace hincapié en que no colabora con cadenas) en distintos barrios según su criterio de lo que es cool. Para ello elaboran ma- 
pas donde muestran, a su criterio, lo mejor de la Barcelona local: "diseñadores, brunch, moda, cultura de vanguardia, bares, slow food, cafés, propuestas eco, coworkings, y todos los lugares cool que no puedes perderte" según se lee en su folleto.

- Qué tipo de reclamo utilizan los apartamentos de Airbnb ofertados en el barrio, revisando aleatoriamente las ofertas disponibles entre junio, julio, agosto y septiembre, en distintos días de la semana, habiendo consultado un total de catorce casos y comprobando si entre los elementos de atracción se incluían la cercanía tiendas de alquiler de bici o la facilidad de ir en bici por la ciudad/barrio.

\section{ALGUNOS DATOS DEL BARRIO}

Según los datos del año 2016, la Renta Familiar Disponible era de 34.127,35 €, ligeramente superior a la media de la ciudad $(32.315,39 €)$. Los otros indicadores sociales asociados a la renta también muestran el carácter medio de Sant Antoni en referencia al conjunto de Barcelona. Si nos fijamos en cuatro indicadores indirectos que nos permiten identificar las características socioeconómicas del barrio podemos observar que:

- $\quad$ Es un barrio densamente poblado, con casi $48.000 \mathrm{hab} / \mathrm{km}^{2}$, el séptimo con una mayor densidad de la ciudad aunque con importantes disparidades internas según secciones censales.

- $\quad$ Presenta una tasa de población con estudios superiores (sobre la población de más de 24 años) ligeramente superior a la media de la ciudad (38,5\% frente a 34,1\%). También, se pueden apreciar las diferencias entre secciones censales dentro del barrio, ya que la población con estudios superiores se concentra a lo largo de la Gran Via y en la Ronda Sant Antoni, mientras que en las secciones más cercanas a Paral·lel y a la zona baja del Raval el porcentaje de población con estudios superiores es menor. La oscilación intrabarrio se sitúa entre $47,8 \%$ y el $30,0 \%$

- $\quad$ El valor catastral de los locales del barrio es $802 € 7 \mathrm{~m}^{2}$, ligeramente superior a la media de la ciudad $\left(795,3 € / \mathrm{m}^{2}\right)$. A pesar de las diferencias internas (entre los 742 y los $906 € / \mathrm{m}^{2}$ ) el barrio constituye unidad más o menos homogénea respecto a los barrios aledaños: mientras en el Raval los valores catastrales son mucho más bajos, en Poble-sec algo más bajos en el Eixample son claramente más altos.

- $\quad$ Si se observan los precios de venta de viviendas de segunda mano, en Sant Antoni el $\mathrm{m}^{2}$ estaba en 2017 a $4591 €$ según datos municipales. Esto sitúa al barrio por encima de la media del conjunto municipal $4284 € / \mathrm{m}^{2}$, pero no entre los más caros (en el ranking se encuentra en la posición 14). La situación cambia si nos fijamos en el incremento porcentual de los precios de venta de la vivienda de segunda mano entre los años 2013 y 2017. Según este indicador, sólo Poble-sec ha tenido un aumento mayor de los precios $(57,6 \%)$ seguido de muy cerca por Sant Antoni $(56,9 \%)$. Y por detrás está el Raval $(54,13)$. Por tanto, el mayor incremento se da en tres barrios contiguos, superior a la media de Barcelona (41,9\%).

En cuanto a los datos sobre movilidad, podemos ver:

- $\quad$ El índice de motorización del barrio es inferior al del conjunto de Barcelona (306,5 vehículos por mil habitantes y 356,6 respectivamente). Fijándonos en el interior del barrio, volvemos a encontrar una mayor tasa de motorización en la zona más próxima a Gran Vía.

- En cuanto a la relación entre los automóviles de mayor potencia (más de 16 caballos fiscales) en relación al total de automóviles, Sant Antoni está en la media municipal $(8,6)$. También en esta ocasión podemos ver desigualdades internas: una vez más, la menor proporción de vehículos más potentes se encuentra en la cercanía de la parte baja del Raval, mientras que en los aledaños a Gran Via la proporción de estos automóviles es más alta. El barrio cuenta con 14 estaciones de bicicleta pública (Bicing). Además de estas, también incluyen parte del barrio otras estaciones fuera de él pero en los límites. En total, el 42,24\% de la superficie del barrio tiene una estación de Bicing a menos de 100 metros y esta cifra alcanza el 74,54\% si la distancia la elevamos hasta los 150 metros (un par de minutos andando). Por lo que respecta a su localización, se pueden distinguir distintas zonas según la intensidad de estaciones. Por un lado, las que se ubican en las avenidas limítrofes del barrio, especialmente en su área central. Por otro lado, la concentración de estaciones en torno al mercado. Una tercera área se localiza en las cercanías de la plaza Universitat, una zona más alejada del corazón del barrio. Finalmente, la zona del barrio centrada en la avenida Mistral es donde menos estaciones hay y la que queda menos servida (es la gran área que está a más de 150 metros de cualquier estación). 


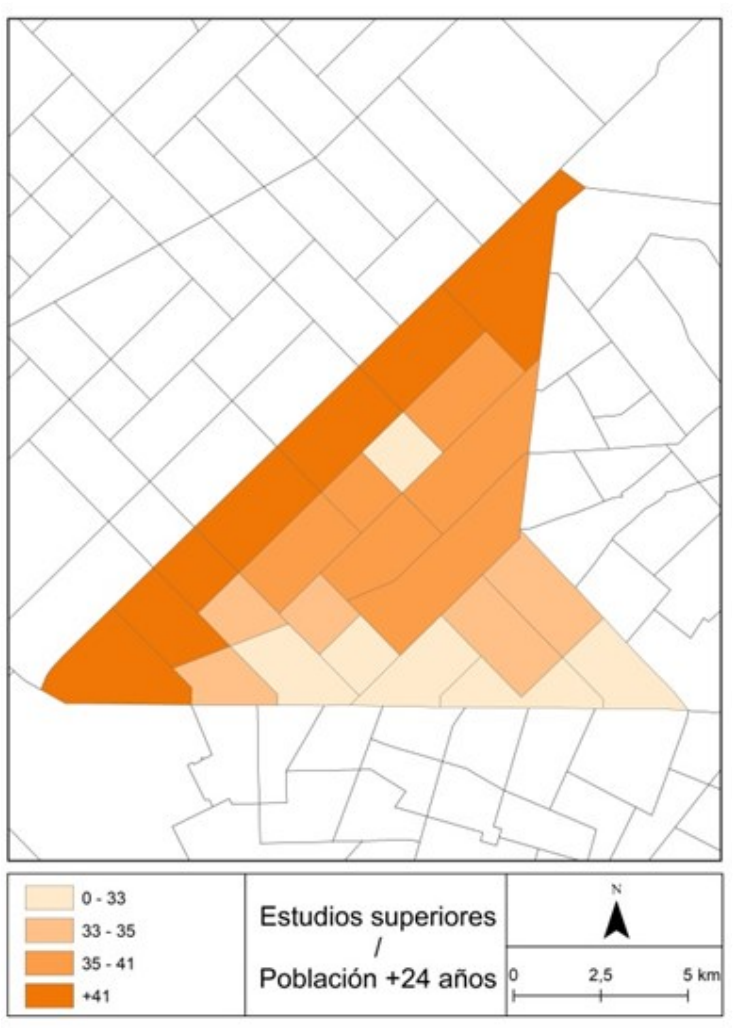

Figura 2.

Fuente: Elaboración propia.

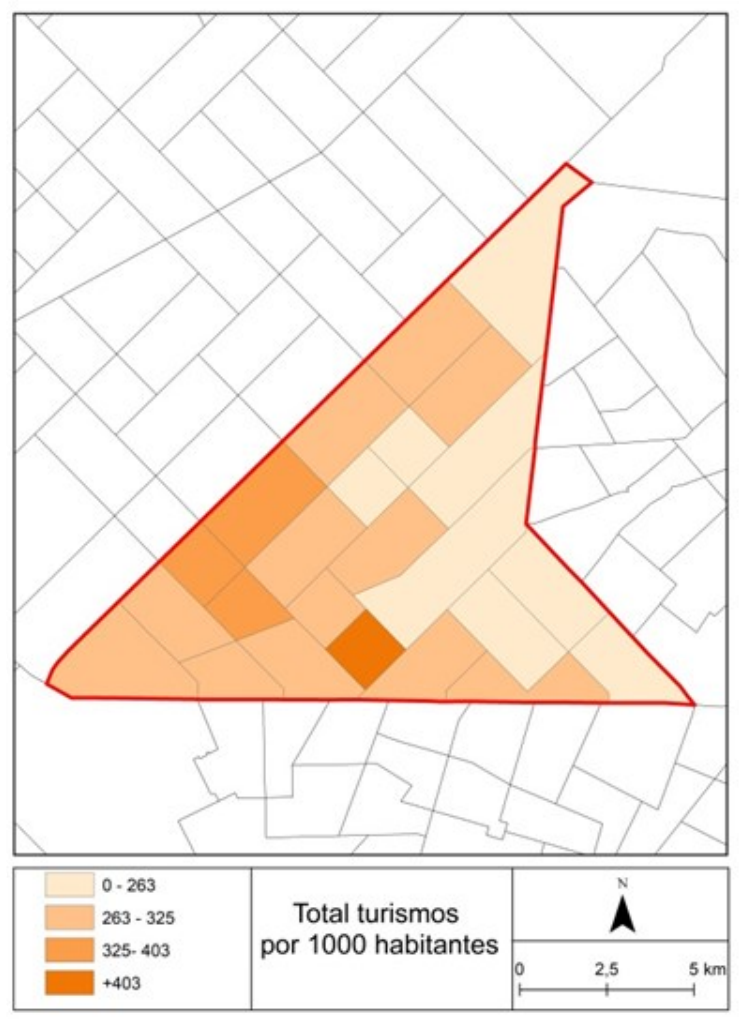

Figura 4.

Fuente: Elaboración propia.

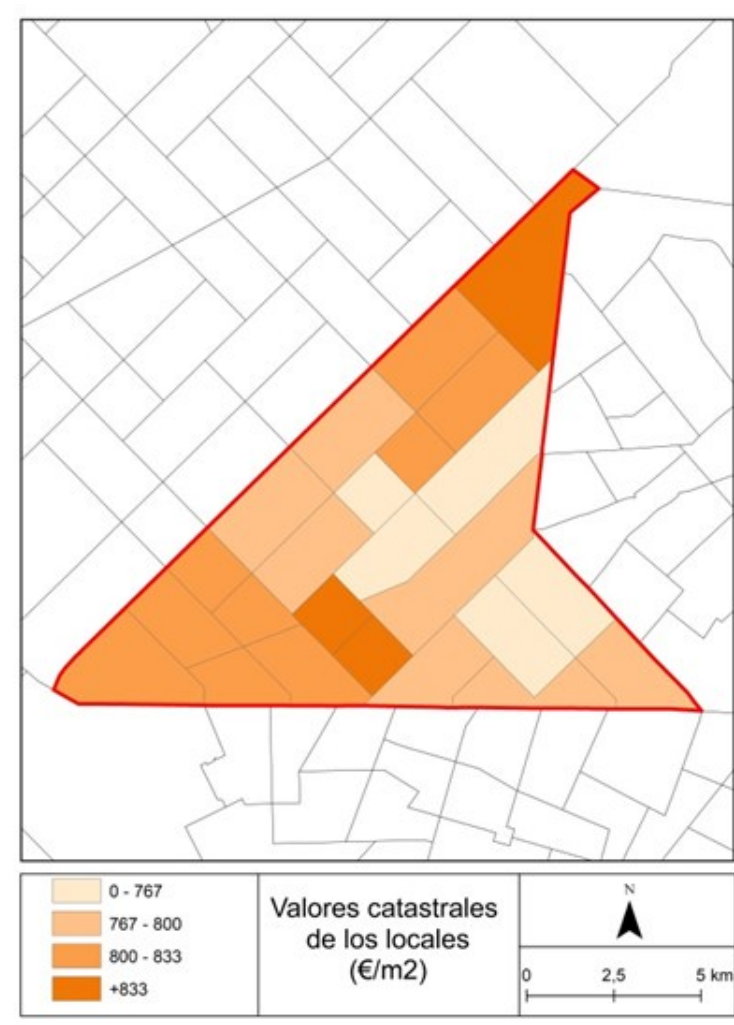

Figura 3.

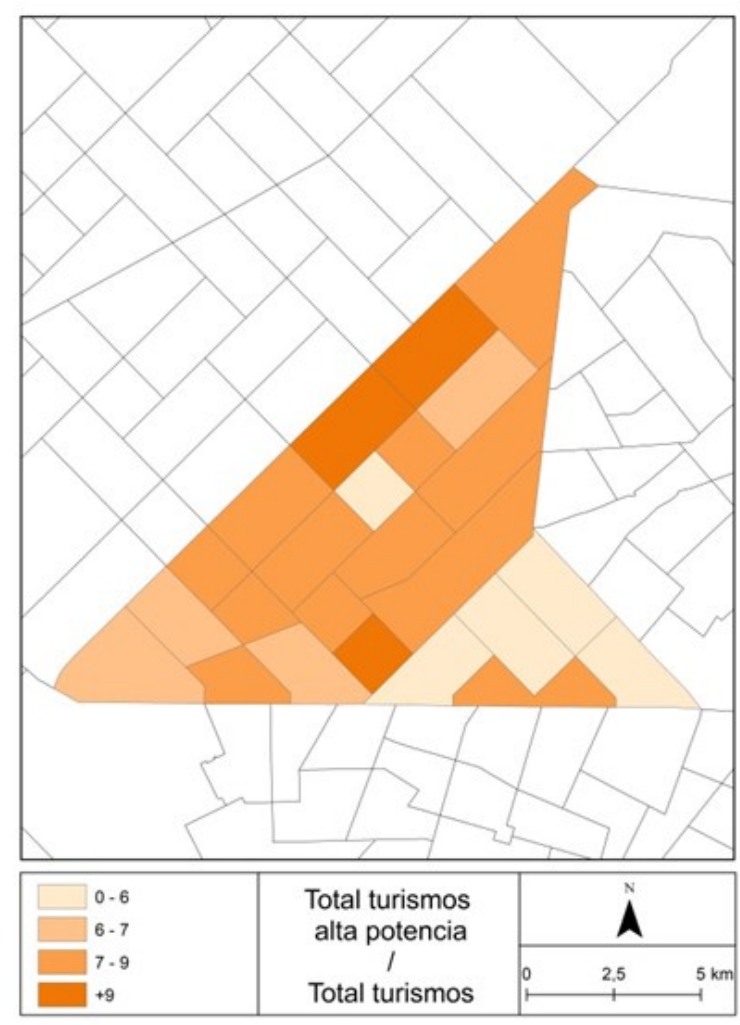

Figura 5. 


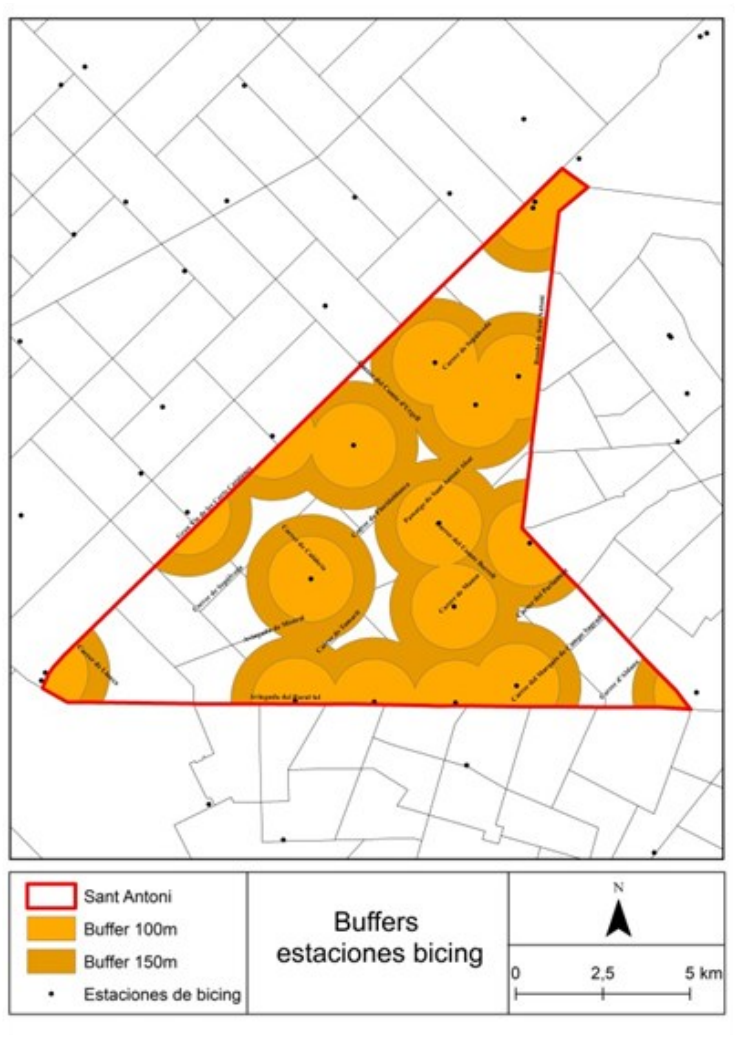

Figura 5.

Fuente: Elaboración propia.

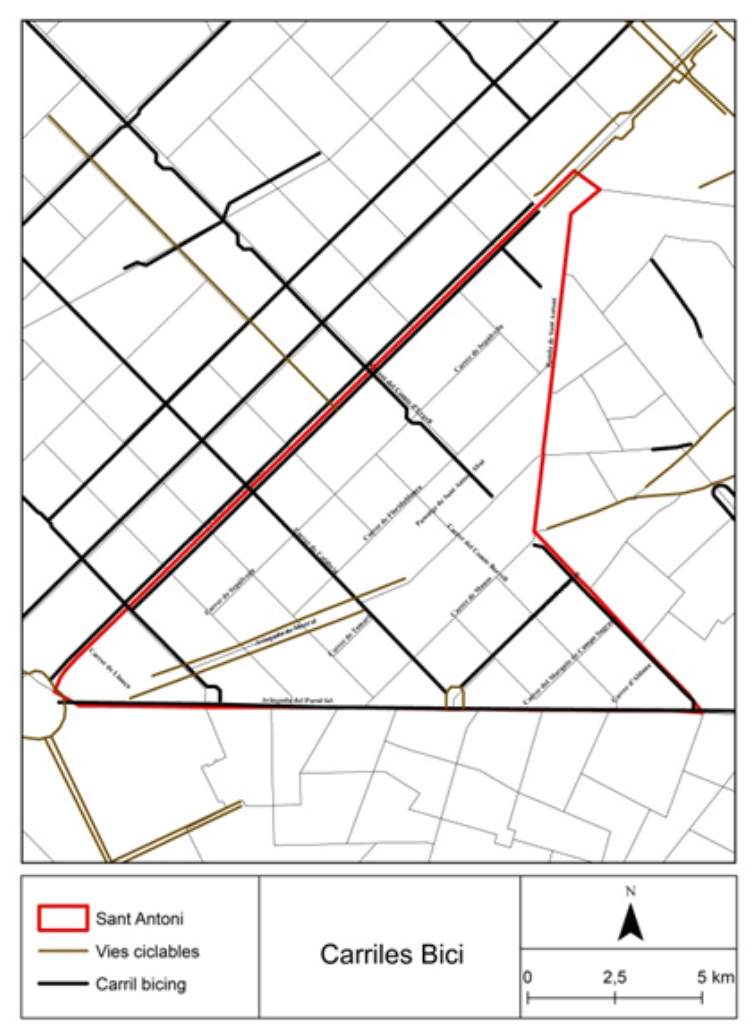

Figura 6

Con respecto a la infraestructura ciclable, el barrio cuenta con distintos carriles bici. Por un lado, encontramos esta infraestructura en las dos grandes avenidas limítrofes (Paral·lel y Gran Via) y además en algunas calles "interiores". En concreto, en NO- SE, encontramos carriles bici cada tres vías. En el sentido transversal, solo existe un carril en la parte baja del barrio. Además de estos carriles, el Ayuntamiento considera como vía ciclable la avenida Mistral. Con la inauguración de la superilla de Sant Antoni, se incrementará el espacio ciclable del barrio.

A pesar de la oferta de infraestructuras para bicicleta en el barrio, se debe tener en cuenta que la apuesta de la ciudad por este modo tiene antecedentes anteriores a lo que se considera el pistoletazo de salida del proceso de gentrificación en Sant Antoni. Aunque la construcción del primer carril bici en la ciudad data de 1990, no es hasta principios del siglo XXI cuando desde el ayuntamiento se impulsa una política de inversión pública en este tipo de infraestructura para el conjunto de la ciudad (o al menos para el conjunto del ensanche). Pero sin duda, el punto de inflexión es la inauguración del sistema de bicicleta pública en 2007. Es en ese momento cuando la bicicleta se incorpora en el imaginario como un elemento más del paisaje urbano. En este sentido, la red de infraestructura bici en la ciudad (sumando carriles y estaciones) rebasa claramente el ámbito del barrio de estudio, que si bien es cierto que cuenta con esta infraestructura, también existe en otros muchos otros barrios de la ciudad. En todo caso, la bicicleta no es un elemento singular de Sant Antoni, aunque también es de Sant Antoni y no deja de ostentar el símbolo cosmopolita, ecológico y cool.

\section{MODERNIZACIÓN Y CAMBIO CULTURAL: LA BICICLETA COMO RECLAMO EN LOS NEGOCIOS DE SANT ANTONI}

En Sant Antoni hay un total de cuatro tiendas de venta de bicicletas, de las cuales se han podido visitar tres. Frente a una que mantiene un carácter deportivo y especializado en ciclismo de montaña, las otras dos presentan un carácter mucho más urbano que se refleja en una estética minimalista y pensada para un barrio de carácter moderno, incluyendo mensajes en inglés destinados a los clientes internacionales. La estética de dichos locales 
encuentra su similitud en un estilo que el periodista Kyle Chayka, de The Guardian, ha bautizado como AirSpace, relacionándolo con los no-lugares de Augé. Según la crítica del periodista, este estilo de decoración de interior basado en el uso mobiliario industrial, maderas claras trabajadas toscamente, bombillas Edison y grandes ventanales destinados a crear interiores más confortables, se extiende globalmente entre los barrios más gentrificados y afecta tanto a bares, restaurantes, oficinas de start-ups, espacios de co-working y a edificios de Airbnb. La manera en la que se globaliza es distinta a las grandes marcas, y no hay una imposición de la cadena para marcar una decoración determinada, sino que es por el uso de las redes sociales por lo que se genera esta homogeneización estética (Chayka, 2016).

Estas dos tiendas de bicicleta de estética -según Chayka- Airspace, son pequeños comercios que aparecieron hace cuatro o cinco años y ambos tienen influencia europea. Corresponden con una época en la que Sant Antoni comenzaba a cambiar el carácter de sus negocios, especialmente por la zona de la calle Parlament, y empezaban a aflorar intentos de remodernizar -quizás con cierto aire posh (pijo, sofisticado)- para darle otro tipo de vida al barrio. Un empuje que venía acompañado por la creación de galerías de arte y preocupación estética de los nuevos locales. Aquel cambio que parecía que seguiría en aumento, ha visto una gran recesión porque a día de hoy los negocios no pueden hacerse cargo de la subida de precio de los alquileres comerciales. La expectación -y la expectativa de muchos propietarios de locales esperando para alquilar- se dirige hacia la apertura del mercado.

El producto a la venta de estos negocios recae mayormente en las bicicletas vintage, llegando incluso a tener técnicos especializados en reparación de las mismas. Estas bicicletas, otrora destinadas a coleccionistas, se han abierto camino entre los ciclistas urbanos que optan por la reutilización de las mismas, en palabras de un vendedor, "por ser consumidores de buen gusto" (entrevista exploratoria, 13/04/2018). Aunque parece que la falta de consolidación del cambio en el perfil de vecino-consumidor ha hecho que disminuya el interés por dichas bicicletas. En su lugar, algunos comercios optan por el alquiler, incluyendo al cliente extranjero con largas estancias. Además de las tiendas visitadas (aunque no todas lo incluyen), hay dos comercios más dedicados exclusivamente al alquiler.

Para profundizar más sobre las posibilidades de alquiler, se han consultado los mapas de disponibilidad de la plataforma Donkey Republic. Donkey Republic es una empresa danesa de alquiler de bicicletas que ofrece la posibilidad de recoger y soltar el vehículo con una mera operación a través de su App, sin preocupación de candados externos o de infraestructura para aparcarlas. No hay horarios ni hay que volver a la tradicional tienda para devolverla. El modus operandis de esta empresa desató las críticas de diferentes sectores, incluidos la Asociación de Bicitours de Barcelona, por utilizar los aparcamientos públicos como almacén de bicicletas (dado que no existe una sede física de almacenamiento y recogida diaria de las mismas) (Franch, 2017). Se ha comprobado que, según lo que afirma la empresa, realizan un control exhaustivo del emplazamiento de sus bicicletas ofertando puntos fijos de recogida/estacionamiento de bicicleta. Mientras que barrios del núcleo central de Barcelona (Raval o Gótico) se mantienen prácticamente libres de estas bicicletas, en Sant Antoni existe una permeabilización entre las calles Manso y Parlament (que tanto por las observaciones de campo como por las entrevistas exploratorias, se muestra como el punto neurálgico del cambio a nivel de comercios en los últimos cinco años). El número de bicicletas disponibles en el barrio oscila entre ocho y trece. En una panorámica más general se observa mucha más concentración de bicicletas en las calles generales que rodean al barrio.

La revisión de la existencia de locales de moda en el barrio vinculados a la bicicleta a través de la plataforma The Trend Map por categorías nos ha permitido observar en qué medida la bicicleta se utiliza como reclamo en este mundo de lo moderno y en qué medida estos bares se encontraban en Sant Antoni. Existen once negocios distribuidos entre el Born, Gòtic y Raval, y uno en Esquerra de l'Eixample, que se proponen como bikefriendly (lugares donde la bicicleta es bien recibida) o vinculados a la bicicleta. En el interior de la trama de Sant Antoni no encontramos ninguno específico de bicicleta, aunque sí que constan diferentes locales calificados como trendy, que van desde gastrobares, como tiendas de ropa o decoración, así como locales de copas. Estos negocios tienen una distribución más concentrada hacia el barrio del Raval, y más concretamente en la calle Parlament.

Por último, atendiendo a la controversia generada por el impacto de la plataforma Airbnb, se ha realizado un rastreo exploratorio en diferentes ofertas de pisos de alquiler comprobando incluyen la bicicleta como un reclamo del propio apartamento o del entorno del barrio. La plataforma Fem Sant Antoni sitúa en 904 las ofertas de alquileres Airbnb en el barrio (además de veintidós agencias inmobiliarias, doce edificios enteros de pisos turísticos, doce hoteles, dieciocho hostales y albergues, y seis edificios completos en venta o ya vendidos como fondos de inversión). A través de la revisión sólo se ha encontrado un caso en el que la bicicleta se utilice como reclamo. 


\section{PRIMERAS CONCLUSIONES PRELIMINARES}

Sant Antoni, a tenor de los datos sociales analizados, es un barrio con características sociales muy próximas a la media de la ciudad. Si nos fijamos a nivel interno, sí que se pueden observar unos indicadores menos favorecidos en la zona del barrio encajada entre el Paral·lel y el Raval, coincidiendo con el espacio en el cual ha saltado la alarma por el proceso de transformación social de los últimos años y cuyo epicentro está en la calle Parlament. Otro indicador de cambio en el barrio lo encontramos en la evolución de precios en el mercado de vivienda de segunda mano puesto que es, junto con el vecino Poble-sec donde más ha aumentado.

El acercamiento a la zona de estudio ha permitido, en primer lugar, establecer que, a diferencia con otros casos observados a nivel internacional -mayormente norteamericano-, la inversión en infraestructura bici no es el motor del proceso de gentrificación que vive el barrio. La transformación -impulsada por la vorágine de un cambio global- que a día de hoy se ve y se ha visto consolidada en diferentes barrios de Barcelona, se maneja en un plano mucho más difuminado que la relación causa-efecto de la construcción de un carril bici. Hablamos de un aumento del turismo en la ciudad hasta niveles antes no alcanzados, de la movilidad y flexibilidad laboral de los nuevos trabajos deslocalizados que permiten trabajar a distancia desde Barcelona o cualquier otra ciudad global, un aumento desmedido del interés del capital internacional por la inversión en la compra de viviendas, y más concretamente del interés por las propiedades verticales, y finalmente, como resultado de estas dinámicas, una crisis de la vivienda cada vez más agudizada.

Sobre esta base, la indagación del papel de la bicicleta en el proceso de cambio social y urbano de Sant Antoni ha girado, por un lado, en un plano más abstracto centrado en el imaginario social y en el papel de la bicicleta como símbolo del cambio, y por otro, en un plano material vinculado a las nuevas tipologías de uso y consumo de la bicicleta que pueden surgir en esta transformación.

La trayectoria observada ya insinúa un cambio en el perfil sociodemográfico de los habitantes del barrio. Este cambio se está observando, paralelamente, en una modificación del carácter de los locales que se evidencia por una estética que tampoco escapa de la globalización. La bicicleta puede convertirse en un símbolo que se inserta en esa revalorización de la estética cosmopolita y preocupada por la sostenibilidad. Esto podría traducirse en una inclinación hacia la posibilidad de los desplazamientos en bicicleta.

El cambio hacia la estética de lo cosmopolita, se ha visto reflejado en un aumento por el interés sobre las bicicletas vintage. La elección de este tipo de bicicletas prioriza lo estético por encima de la funcionalidad. En el caso de Sant Antoni, las tiendas destinadas al ciclismo urbano ofertan este tipo de producto entendiendo que la posibilidad de venta está relacionada con el atractivo de barrio moderno, y por tanto, la aparición de un nuevo perfil de cliente.

Pero no es el único. La llamada de estas tiendas de bicicleta se expande hacia el cliente internacional, que encuentra negocios adaptados al inglés -entre otros idiomas- en los que se ofertan nuevos modelos de intercambio. Es el caso de los residentes de estancias de medio plazo en Barcelona, a los que se le ofrece la posibilidad de comprar la bicicleta y re-venderla en la misma tienda cuando llegue el momento de marchar. A través del boca a boca aparece un nuevo flujo de clientes que se va consolidando.

Por último, se ha observado que tanto la posibilidad del alquiler como de la reparación, abre la relación con los nuevos modelos de negocio de reparto en bicicleta. Los repartidores/as de estos negocios dependen de su propia bicicleta para cumplir con las entregas, y dada la centralidad del barrio encuentran en estas tiendas un punto seguro donde poder dejar la bici en reparación y alquilar otra mientras tanto.

Si bien en la escala de barrio, y según el estudio exploratorio, no se ha percibido una relación directa de la bicicleta con los negocios que se encuentran vinculados a la plataforma The Trend Map, sí que en los barrios circundantes se observa la existencia de la bicicleta, tanto en la decoración como en estrategias de mercado (por ejemplo el concepto bicifriendly) como un reclamo que se vincula a un estilo de vida moderno y saludable.

En cuanto a la relación con el turismo, no se ha observado que en el momento actual Sant Antoni utilice como reclamo la bicicleta o resulte especialmente atractivo para este tipo de uso. Sin embargo, estos resultados son preliminares. Con la apertura del mercado y la superilla se prevé que este proceso se acelere y Sant Antoni, siendo un enclave entre Montjuic y la zona más turística del Gòtic y Born, se convierta en un barrio mucho más atractivo para el uso de la bicicleta -también para los turistas-. Tras la consolidación de las intervenciones urbanísticas que están concluyendo en la actualidad (mayo de 2018), sería oportuno continuar con un análisis más profundo sobre el proceso dialéctico entre los cambios sociodemográficos del barrio y su materialización en las formas de uso y consumo de la bicicleta. 


\section{AGRADECIMIENTOS}

Esta investigación se lleva a cabo con la ayuda financiera de los proyectos 2017FI_B1118 de la Generalitat de Catalunya y CSO2016-74888-C4-2-R del Ministerio de Educación y Ciencia.

\section{BIBLIOGRAFÍA}

AJUNTAMENT DE BARCELONA (sin fecha). El programa Superilles a Sant Antoni, la transformació en marxa. Barcelona, España: Ajuntament de Barcelona. Recuperado de: http://ajuntament.barcelona.cat/superilles/ca/superilla/sant-antoni. [consultado: 16/04/2018]

CHAYKA, K. (2016). Same old, same old. How the hipster aesthetic is taking over the world. The Guardian (7 de agosto de 2016). Recuperado de: https://www.theguardian.com/commentisfree/2016/aug/06/hipster-aesthetic-taking-over-world. [consultado: 24/04/2018]

DANYLUK, M., y LEY, D. (2007). Modalities of the New Middle Class : Ideology and Behaviour in the Journey to Work from Gentrified Neighbourhoods in Canada. Urban Studies, 44(11), 2195-2210.

FEM SANT ANTONI (5 de octubre 2017). El mapa de la gentrificació: carrer Parlament. Barcelona, España: Fem Sant Antoni. Recuperado de: https://femsantantoni.wordpress.com/2017/10/05/el-mapa-de-la-gentrificacio-carrer-parlament/. [consultado: 12/04/2018]

FLANAGAN, E., LACHAPELLE, U., y EL-GENEIDY, A. (2016). Research in Transportation Economics Riding tandem: Does cycling infrastructure investment mirror gentri fi cation and privilege in Portland OR and Chicago , IL? Research in Transportation Economics, 60, 14-24.

FrANCH, S. (2017). El desembarco de las bicis de alquiler 'online' en Barcelona pone en pie de guerra al sector (28 de junio de 2017). Recuperado de: https://www.eldiario.es/catalunya/barcelona/desembarco-alquiler-online-Barcelonaguerra_0_659334609.html. [consultado: 27/04/2018]

GIBSON, T. A. (2015). The rise and fall of Adrian Fenty, Mayor-Triathlete: cycling, gentrification and class politics in Washington DC. Leisure Studies, 34(2), 230-249.

LUGO, A. E. (2012). Can human infrastructure combat green gentrification?: ethnographic research on bicycling in Los Angeles and Seattle. In M. Isenhour, Cindy; McDonogh, Gary; Checker (Ed.), Sustainability in the Global City: Myth and Practice (pp. 306-328). New York: Cambridge University Press

SMITH, N. (2002). New Globalism, New Urbanism : Gentrification as Global Urban Strategy. Antipode, 34(3), 427-450.

STEHLIN, J. (2015). Cycles of investment : bicycle infrastructure, gentrification, and the restructuring of the San Francisco Bay Area. Environment and Planning A, 47, 121-137.

STEIN, B. S. (2011). Bike Lanes and Gentrification: New York City's Shades of Green. Progressive Planning, $188,34-37$.

THE TREND MAP (sin fecha). ¿Qué es trend map?. Barcelona, España: The trend map. Recuperado de: http://trendmap.es/es/ que-es/ [consultado: 04/05/2018]

TIME OUT. (2017). Els millors bars del carrer Parlament. Barcelona, España: Time Out. (27 de febrero 2017)Recuperado de: https://www.timeout.cat/barcelona/ca/bars-pubs/els-millors-bars-del-carrer-parlament. [consultado: 02/05/2018]

VITH, T. A., y MÖSSNER, S. (2017). Contesting sustainable transportation : bicycle mobility in Boston and beyond. Journal of the Geographical Society of Berlin, 148(4), 229-237. 